\title{
Biopolymer Based Nanoparticles: Isolation, Characterization, and Applications ${ }^{\dagger}$
}

\author{
J. Lavanya Agnes Angalene 1,*, S.M. Roshini ${ }^{2}$ \\ 1 Department of Biotechnology, School of Biosciences and Technology, Vellore Institute of Technology, Vellore 632014 \\ Tamil Nadu, India \\ 2 Department of Biotechnology, School of Bio and Chemical Engineering. Sathyabama Institute of Science and Technology, \\ Chennai 600119 Tamil Nadu, India \\ * Correspondence: lavanyaagnes97@gmail.com; \\ $\dagger$ Presented at International e-Conference on Bioengineering for Health and Environment (ICBHE 2020)
}

Received: 5.07.2020; Revised: 10.07.2020; Accepted: 12.07.2020; Published: 15.07.2020

\begin{abstract}
The budding field of Nanotechnology has become one of the most popular areas of interest for current research and development, especially in the research areas of polymer science and technology. Natural Polysaccharide polymers are found abundant in nature and are now frequently been used for the preparation of many nanomaterials. When compared to the synthetic polymers available, natural biopolymers are more biocompatible, biodegradable, and renewable in nature. The production of Nanomaterials from biopolymers has eventually improved the properties of the Nano-products developed. Nature has provided many different varieties of natural polymers; one such plant-derived polymer is the plant Gum exudate. Gums are commonly exuded by plants at very small quantities, forming conspicuous incrustations. These Natural gums exudates are polysaccharides. They are chemically inert, biocompatible, biodegradable, and sustainable in nature. In this study, plant gumbased biopolymers were used for the synthesis of nanoparticles. The natural gum exudates were collected from the plants' Araucaria heterophylla, Azadirachta indica. The obtained biopolymers were purified by the solvent extraction method and were characterized by UV-Vis Spectroscopy, FTIR, and TGA. Further, the purified biopolymers were subjected to the encapsulation of drugs (as Nanocarrier) and coating for SPIONs (superparamagnetic iron oxide nanoparticles). The synthesized nanoparticles were characterized by UV-Vis Spectroscopy, FTIR, SEM, EDAX, TEM, and AFM. The biopolymerbased nanocarriers were about 200nm in size and were utilized for effective drug delivery studies using MCF-7 Breast cancer lines. Finally, the biopolymer encapsulated SPIONs particles of size around 40nm were used for water treatment studies - removing the heavy metal chromium as well as mosquito larvae from water. Thus, the nano-products developed were found to be more eco-friendly, efficient, and also cost-effective.
\end{abstract}

Keywords: Biopolymers; Solvent extraction; Polysaccharide; SPIONs; Encapsulation; SEM; TEM;

AFM; FTIR; UV-Vis Spectroscopy.

(C) 2020 by the authors. This article is an open-access article distributed under the terms and conditions of the Creative Commons Attribution (CC BY) license (https://creativecommons.org/licenses/by/4.0/).

\section{Funding}

This research received no external funding.

\section{Acknowledgments}

This research has no acknowledgment.

\section{Conflicts of Interest}

The authors declare no conflict of interest. 